\title{
Comparison of two misoprostol regimens for cervical priming before surgical pregnancy termination at 13 to 16 weeks gestations
}

\author{
Dennis G. Chambers, Robin J. Willcourt, Anthony R. Laver, Jane K. Baird, Wye Y. Herbert
}

The Queen Elizabeth Hospital Pregnancy Advisory Centre, Woodville Park, Adelaide, Australia.

Email:dennis.chambers@health.sa.gov.au

Received 14 October 2011; revised 19 November 2011; accepted 4 December 2011.

\begin{abstract}
Background: The optimal regimen has still to be determined for the use of misoprostol in the surgical termination of pregnancy in the early second trimester. Objective: To compare the outcomes of two different regimens for cervical priming with misoprostol before dilatation and evacuation ( $D \& E$ ) in 13 weeks - 16 weeks gestation pregnancy terminations. Methods: A retrospective analysis was performed of the medical records of two cohorts of 334 women each who were treated with either 3 sublingual doses of 2 misoprostol $200 \mu$ g tablets 30 minutes apart on admission or the same dosage preceded by 1 oral tablet of misoprostol $200 \mu \mathrm{g}$ at home 3 hours before admission. Results: The addition of the home tablet of misoprostol increased the rate of one day completion of $D$ \& $E$ from $97.3 \%$ to $100 \%(P=0.004)$, and the overall mean theatre time for $D \& E$ was reduced by $12.3 \%$ in parous women $(P=0.001)$ and $6.4 \%$ in nulliparous women $(P=0.003)$ with the reduction being consistent across all gestations. Conclusions: This retrospective study showed that the addition of 1 oral tablet of misoprostol $200 \mathrm{\mu g}$ at home 3 hours before admission to a regimen of 3 sublingual doses of 2 misoprostol $200 \mu$ g tablets 30 minutes apart on admission significantly increases the probability of all women at 13 weeks - 16 weeks gestation completing a termination of pregnancy in one day with a single $D \&$ E procedure and with a reduced theatre time.
\end{abstract}

Keywords: Early Second-Trimester Surgical Abortion; D \& E; Misoprostol; Cervical Priming

\section{INTRODUCTION}

The Pregnancy Advisory Centre (PAC) is a government funded day-surgery clinic providing over 2500 surgical and medical terminations of pregnancy (TOP) each year in South Australia. Second trimester surgical termination of pregnancies up to 22 weeks gestation have been performed in this clinic using misoprostol and osmotic cervical dilators where necessary since 1994 . The incidence of these terminations has remained constant over this period with approximately $9 \%$ of all terminations occurring at 13 weeks - 16 weeks gestation, an incidence in line with published figures overseas [1]. Although most women in our clinic at 13 weeks - 16 weeks gestation have been terminated in one day after cervical priming with 3 sublingual doses of 2 misoprostol $200 \mu$ g tablets 30 minutes apart, with dilatation and evacuation (D \& E) 3 hours after the last dose, we have found that approximately $3 \%$ of women could not be adequately cervically dilated in one day with this regimen because they were very poor responders to misoprostol. Therefore in December 2009 the clinic introduced the addition to this regimen of a single oral tablet of misoprostol $200 \mu \mathrm{g}$ taken at home 3 hours before admission. Oral administration was chosen to avoid distressing cramping, bleeding and nausea that occur more frequently following sublingual administration [2]. Because there is little published data on the outcomes of different cervical priming regimens before D \& $\mathrm{E}$ at 13 weeks - 16 weeks we decided to research the outcomes in our clinic of the two misoprostol regimens.

\section{MATERIALS AND METHODS}

The study design was a retrospective review of the clinical records of two cohorts of consecutive cases of women undergoing surgical termination of pregnancy at 13 to 16 weeks gestation. This clinical audit study conformed to the standards required for Australian National Health and Medical Research Council exclusion for ethics approval. The first cohort of 334 women was treated with 3 sublingual doses of 2 misoprostol $200 \mu$ g tablets administered 30 minutes apart on admission. The second cohort of 334 women was treated with the same dosage after admission preceded by 1 oral tablet of misoprostol 
$200 \mu \mathrm{g}$ at home 3 hours before admission. The dosage after admission of 6 misoprostol tablets totaling $1200 \mu \mathrm{g}$ is the dosage we have used for approximately the last 10 years. This is the optimal dosage that we have found achieves adequate cervical priming prior to D \& E without adverse effects; it was determined by incremental increases over several years of study [3].

In both cohorts three hours after the last dose of misoprostol the woman was taken to theatre for D \& E. All D \& E procedures at our clinic are performed under intravenous propofol anesthesia with the addition of an intracervical block of lignocaine local anesthetic with adrenaline. The cervix is dilated with rigid Hawkin-Ambler dilators to a dilatation equal to the number of gestational weeks in millimeters (mm). When the cervical dilatation and softening the misoprostol priming of the cervix has achieved is adequate the range of rigid dilatation required is $0 \mathrm{~mm}-3 \mathrm{~mm}$ and force is never used. A previous study from this clinic has demonstrated a connection between forceful dilatation and perforation of the uterus [4]. If the target dilatation of the weeks gestation in $\mathrm{mm}$ cannot be achieved without force further cervical priming with osmotic dilators and misoprostol is used. Routine intra-operative ultrasound guidance is used allowing the operator to see instruments in the uterus and direct them to the fetal parts safely.

After the use of the additional home tablet of misoprostol in 334 early second trimester women we decided to audit our results, and this number decided the size of the cohorts. Medical records were examined and data was collected relating to parity, gestation, the number of procedures, the number of days of treatment, the theatre time for each woman at D \& E, and complications in each cohort. The theatre times were used rather than operation times as these was collected with more exact precision, the time of entry into and exit from the operating theatre being collected and recorded separately by both the anesthetist and theatre nurse with cross checking; the time of entry was the time at which the woman was wheeled into the operating theatre from the anesthetic room, and the exit time was the time she was wheeled out of the theatre into the recovery room. Transfer times onto and off the operating table were the same for all women and as all instruments used were in prepacked and presterilized bundles there were no sterilization delays. We also surveyed the incidence of side effects experienced by the 334 women taking misoprostol $200 \mu \mathrm{g}$ at home 3 hours before admission. On admission these women completed a questionnaire in which they self assessed the severity-mild, moderate, or severeof any side effects of cramps, bleeding, nausea or diarrhea. All the procedures analyzed were carried out by the same operators who had many years experience. The statistical analysis was performed using Fisher's exact test.

\section{RESULTS}

In the cohort without home misoprostol there was 1 woman at 13 weeks, 1 woman at 14 weeks and 7 women at 16 weeks gestation who could not be completed in a single stage procedure; inadequate cervical priming at this procedure necessitated further cervical priming and D \& E the next day. In the cohort with a home dose of misoprostol added all women were completed in a one stage D \& E procedure. The addition of the home tablet of misoprostol increased the rate of one day completion of $\mathrm{D}$ \& $\mathrm{E}$ from $97.3 \%$ to $100 \%(P=0.004)$. The theatre times in each cohort for nulliparous women, with a break down by weeks gestation, are shown in Table 1, and the times for parous women with a similar breakdown are shown in Table 2. The overall mean theatre time for D \& E was reduced by $12.3 \%$ in parous women $(P=0.001)$ and $6.4 \%$ in nulliparous women $(P=0.003)$ with the reduction being consistent across all gestations and parities.

Table 1. Nulliparous women D \& E mean theatre times 13 weeks - 16 weeks gestations with and without home misoprostol priming; $\mathrm{n}=$ number at each gestation.

\begin{tabular}{cccccc}
\hline Mean time & 13 weeks gestation & $\mathbf{1 4}$ weeks gestation & $\mathbf{1 5}$ weeks gestation & $\mathbf{1 6}$ weeks gestation & Overall mean time \\
\hline No home misoprostol & 13.2 minutes $\mathrm{n}=59$ & 16.1 minutes $\mathrm{n}=65$ & 16.7 minutes $\mathrm{n}=45$ & 19.4 minutes $\mathrm{n}=17$ & 15.6 minutes $\mathrm{n}=186$ \\
With home misoprostol & 13.1 minutes $\mathrm{n}=66$ & 14.6 minutes $\mathrm{n}=57$ & 16.3 minutes $\mathrm{n}=26$ & 16.5 minutes $\mathrm{n}=26$ & 14.6 minutes $\mathrm{n}=175$ \\
\hline
\end{tabular}

Table 2. Parous women D \& E mean theatre times 13 weeks - 16 weeks gestations with and without home misoprostol priming; $\mathrm{n}=$ number at each gestation.

\begin{tabular}{cccccc}
\hline Mean time & 13 weeks gestation & 14 weeks gestation & 15 weeks gestation & 16 weeks gestation & Overall mean time \\
\hline No home misoprostol & 14.9 minutes $\mathrm{n}=50$ & 15.1 minutes $\mathrm{n}=56$ & 16.5 minutes $\mathrm{n}=36$ & 16.8 minutes $\mathrm{n}=6$ & $\begin{array}{c}15.5 \text { minutes } \\
\mathrm{n}=148\end{array}$ \\
With home msoprostol & 12.4 minutes $\mathrm{n}=53$ & 14.1 minutes $\mathrm{n}=51$ & 14.5 minutes $\mathrm{n}=35$ & 14.1 minutes $\mathrm{n}=20$ & 13.6 minutes $\mathrm{n}=159$ \\
\hline
\end{tabular}




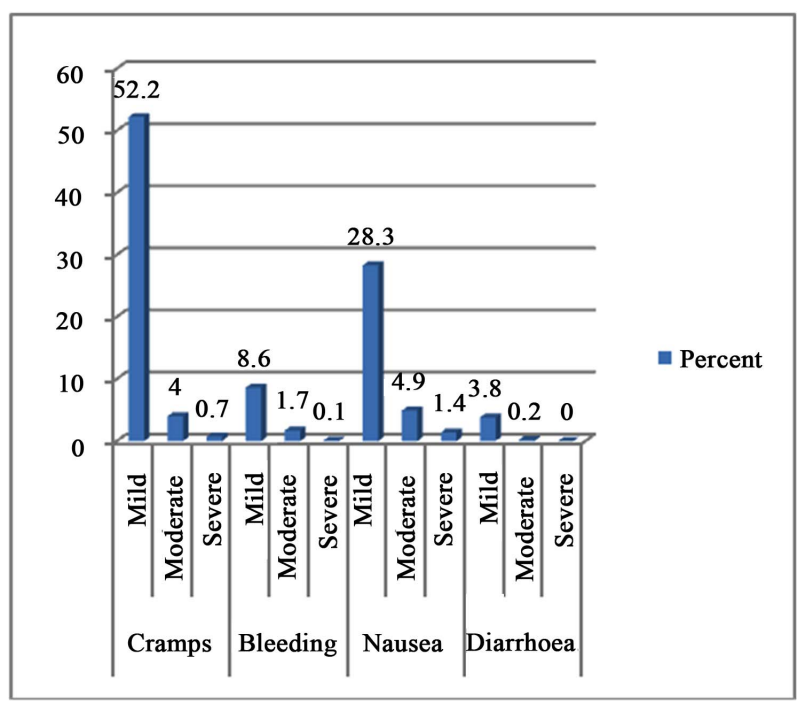

Figure 1. Percentage of women with side effects from one oral tablet of misoprostol $200 \mu \mathrm{g}$ taken at home 3 hours before admission.

The incidence of side effects in 1000 women taking

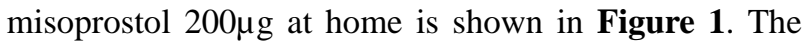
only significant side effect was mild cramping in 52.2\% of women; mild nausea was reported in $28.3 \%$ of women, but this was difficult to separate from pregnancy morning sickness. None of the side effects of the home dose of oral misoprostol $200 \mu \mathrm{g}$ were severe enough to require treatment.

There was one cervical laceration requiring suture at 16 weeks gestation in the cohort without home misoprostol; there was no other uterine trauma, hemorrhage, infection or other serious complication occurring in either cohort. This gave complication rates of $0.3 \%$ for the no home misoprostol and $0.0 \%$ for the home misoprostol cohorts. The demographic makeup of both cohorts was similar including maternal age, parity, and gestational age.

\section{DISCUSSION}

Every year at the PAC clinic in Adelaide, Australia approximately $9 \%$ of the women seeking termination of pregnancy are in the 13 to 16 weeks gestation range; this corresponds with published incidence data from overseas [1]. This early second trimester group provides different challenges to the late second trimester 17 weeks and over gestation group which in the main cannot be safely terminated with misoprostol cervical priming alone, the addition of osmotic cervical dilators usually being required to ensure safe D \& E [5]. In a previous study from this clinic inadequate cervical priming has been determined as increasing the risk of uterine perforation during second trimester D \& E [4]. The misoprostol dosage recommended in the current literature is $400 \mu \mathrm{g}$ vaginally or buccally for cervical priming at 12 to 16 weeks gestation [6]. This is below the dosage level that we have found to be optimally effective and safe, namely 6 to 7 tablets of misoprostol $200 \mu \mathrm{g}$, a total of 1.200 to 1.400 $\mu \mathrm{g}$. With this higher dosage we have never experienced any serious adverse effects; in particular, despite $20 \%$ $25 \%$ of our parous women having a Caesarean scar, we have never seen a uterine rupture in 10 years of this usage. We attribute this to never exceeding a misoprostol dose of $400 \mu \mathrm{g}$ in any 30 minute period, and never using the vaginal route of administration for cervical priming.

Our retrospective study compared two misoprostol dosages in two cohorts of women undergoing $\mathrm{D} \& \mathrm{E}$ termination of pregnancy at 13 to 16 weeks, and demonstrated that the larger dosage, when commenced at home 3 hours before admission, enabled all cases to be completed in one day with no serious complications, the success rate rising from $97.3 \%$ to $100 \%(P=0.004)$. The addition of the home dose also reduced the overall average theatre time for D\&E by $12.3 \%$ in parous women $(P=0.001)$ and $6.4 \%$ in nulliparous women $(P=0.003)$, with the reduction being consistent across all gestations and parities. This reduction in theatre and operation times reflects the consistent greater ease in performing the D \& E procedures due to better cervical priming with the home dose of misoprostol. The improved cervical priming also led to a decreased need for the use of rigid cervical dilators and abolished the need to ever use force with rigid dilators, thus reducing the risk of cervical trauma.

A Cochrane review in 2008 concluded that cervical preparation with osmotic dilators and/or misoprostol before second-trimester D \& E is safe and effective [7]. Cervical preparation with osmotic dilators is an alternative to misoprostol but has the disadvantage of requiring another procedural stage, and there is often a delay overnight before the cervix is sufficiently primed to ensure that the D \& E can be performed safely. A third alternative for cervical priming is the administration of mifepristone $200 \mathrm{mg}$, but this has the disadvantage of higher cost as well as more clinic visits by the patient; also another effect of mifepristone is the possibility of expulsion of the fetus before D \& E, with the consequent risk to the woman's health, as this expulsion may occur at any time and place [8]. A Cochrane Review in 2010 concluded that mifepristone is associated with high rates of pre-procedural expulsions and does not appear to be a useful method of cervical preparation before second-trimester dilation and evacuation [9].

There were no serious complications in either of the two cohorts we have surveyed undergoing D \& E at 13 weeks - 16 weeks; there were 9 women in the cohort that did not receive a misoprostol dose at home who needed an additional 24 hours of cervical priming with osmotic dilators to adequately prepare the cervix for safe D \& E. 
All women receiving a home dose of misoprostol 3 hours before admission had their D \& E completed in one day in a single stage procedure without any complications. Nucatola et al. have reviewed complication rates for misoprostol primed D \& E at 12 weeks - 16 weeks, and for their series they recorded a perforation rate of $0.045 \%$ which they determined was in line with rates previously reported in the literature [10].

The same doctors performed all the operations in both cohorts and all operators were very experienced at the beginning of the study period and unlikely to have gained any relevant increase in experience by its end.

A limitation of the study was the use of theatre times rather than operation times. Reliable operation times were not available as, this being a retrospective study, operation times had not been recorded with the same accuracy as would occur in a prospective study; accurate theatre times were available as our clinic has a protocol in place for precision in the recording of theatre times. We believe the consistency of difference of the theatre times between the two cohorts indicates that they do reliably reflect operation times. It is our opinion that it would have been unethical to conduct a prospective double blind study as we already had firm evidence from our first trimester study of home misoprostol dosage of the significant benefits of this regime [3].

This retrospective study demonstrates that the addition of one oral tablet of misoprostol $200 \mu \mathrm{g}$ at home 3 hours before admission to a regimen of 2 tablets $1 / 2$ hourly for three doses on admission enables $100 \%$ of women at 13 weeks - 16 weeks gestation to complete a termination of pregnancy 3 hours after the last dose of misoprostol, in one day, with a single D \& E procedure, in a shorter time, and with minimal complications. This certainty of outcome is of help in the planning and scheduling of operation lists

\section{ACKNOWLEDGEMENTS}

We are grateful to Dr John Field, Statistician, Faculty of Health Sciences, University of Adelaide for statistical analysis. No author has any conflict of interest.

\section{REFERENCES}

[1] Gamble, S.B., Strauss, L.T., Parker, W.Y., Cook, D.A., Zane, S.B. and Hamdan, S. (2008) Centers for disease control and prevention (CDC). Abortion SurveillanceUnited States 2005. MMWR Surveillance Summaries, 57, 1-32.

[2] Aronsson, A., Bygdeman, M. and Gemzell-Danielsson, K. (2004) Effects of misoprostol on uterine contractility following different routes of administration. Human Reproduction, 19, 81-84. doi:10.1093/humrep/deh005

[3] Chambers, D.G., Mulligan, E.C., Laver, A.R., Weller, B.K., Baird, J.K. and Herbert, W.Y. (2009) Comparison of four perioperative misoprostol regimens for surgical termination of first-trimester pregnancy. International Journal of Gynecology and Obstetrics, 7, 211-215. doi:10.1016/j.ijgo.2009.07.034

[4] Pridmore, B.R. and Chambers, D.G. (1999) Uterine perforation during surgical abortion: Review of diagnosis, management and prevention. Australian and New Zealand Journal of Obstetrics and Gynaecology, 39, 349-353. doi:10.1111/j.1479-828X.1999.tb03413.x

[5] Chambers, D.G., Willcourt, R.J., Laver, A.R., Baird, J.K. and Herbert, W.Y. (2011) Comparison of Dilapan-S and laminaria tents for cervical priming before surgical pregnancy termination at 17 to 22 weeks gestations. International Journal of Women's Health, 3, 347-352. doi:10.2147/IJWH.S25551

[6] Wilson, L.C., Meyn, L.A. and Creinin, M.D. (2011) Cervical preparation for surgical abortion between 12 and 18 weeks of gestation using vaginal misoprostol and Dilapan-S. Contraception, 8, 511-516. doi:10.1016/j.contraception.2010.10.004

[7] Lohr, P.A., Hayes, J.L. and Gemzell-Danielsson, K. (2008) Surgical versus medical methods for second trimester induced abortion. Cochrane Database Systematic Reviews, 1, CD006714.

[8] Carbonell, J.L., Gallego, F.G., Lorente, M.P., Bermudez, S.B., Sala, E.S., Gonzalez, L.V. and Texido, C.S. (2007) Vaginal vs. sublingual misoprostol with mifepristone for cervical priming in second-trimester abortion by dilatation and evacuation: A randomized clinical trial.. Contraception, 75, 230-237. doi:10.1016/j.contraception.2006.11.007

[9] Newman, S.J., Dalve-Endres, A., Diedrich, J.T., Steinauer, J.E., Meckstroth, K. and Drey, E.A. (2010) Cervical preparation for second trimester dilatation and evacuation. Cochrane Database Systematic Reviews, 8, CD007310.

[10] Nucatola, D., Roth, N., Saulsberry, V. and Gatter, M. (2008) Serious adverse events associated with the use of misoprostol alone for cervical preparation prior to early second trimester surgical abortion (12 weeks - 16 weeks).. Contraception, 78, 245-248.

doi:10.1016/j.contraception.2008.04.121 